\title{
Cultivation and Genomic Characterization of the Bile Bacterial Species From Cholecystitis Patients
}

\section{OPEN ACCESS}

Edited by:

Martin Maiers,

National Marrow Donor Program,

United States

Reviewed by:

Babak Haghshenas,

Kermanshah University of Medical

Sciences, Iran

Maryam Tidjani Alou,

Aix Marseille Université, France

*Correspondence:

Yi Xin

jimxin@hotmail.com

Xianhai Mao

maoxianhai@hunnu.edu.cn

Yufang Ma

yufangma@dmu.edu.cn

${ }^{\dagger}$ These authors have contributed equally to this work

Specialty section:

This article was submitted to

Evolutionary and Genomic

Microbiology,

a section of the journal

Frontiers in Microbiology

Received: 11 July 2021

Accepted: 07 October 2021

Published: 01 November 2021

Citation:

Yan Q, Zhang S, Li S, Wang G,

Zhang $A$, Jin T, Zhang $Y, L v Q$, Xiao M, Sun Y, LiX, Cui S, Li R, Ma X, Wang C, Tian X, Duan X, Xin Y, Mao X and Ma $Y$ (2021) Cultivation and Genomic Characterization of the Bile Bacterial Species From

Cholecystitis Patients.

Front. Microbiol. 12:739621. doi: 10.3389/fmicb.2021.739621
Qiulong Yan ${ }^{1,2 t}$, Siyi Zhang ${ }^{1 \dagger}$, Shenghui $i^{3{ }^{3+}}$, Guangyang Wang ${ }^{1,4}$, Aiqin Zhang ${ }^{3}$, Taiyang Jin', Yue Zhang ${ }^{3}$, Qingbo Lv'3 ${ }^{3}$ Manchun Xiao', Yuanyuan Sun', Xiang Li', Song Cui ${ }^{1}$, Rui Li ${ }^{1}$, Xiaochi Ma ${ }^{5}$, Chao Wang ${ }^{5}$, Xiangge Tian ${ }^{5}$, Xiaohui Duan' ${ }^{2}$, Yi Xin ${ }^{4 *}$, Xianhai $\mathrm{MaO}^{2 *}$ and Yufang $\mathrm{Ma}^{1 *}$

${ }^{1}$ Department of Microbiology, College of Basic Medical Sciences, Dalian Medical University, Dalian, China, ${ }^{2}$ Department of Hepatobiliary Surgery, Hunan Provincial People's Hospital, The First Affiliated Hospital of Hunan Normal University, Changsha, China, ${ }^{3}$ Puensum Genetech Institute, Wuhan, China, ${ }^{4}$ Department of Biotechnology, College of Basic Medical Sciences, Dalian Medical University, Dalian, China, ${ }^{5}$ College of Pharmacy, Dalian Medical University, Dalian, China

The microbes in human bile are closely related to gallbladder health and other potential disorders. Although the bile microbial community has been investigated by recent studies using amplicon or metagenomic sequencing technologies, the genomic information of the microbial species resident in bile is rarely reported. Herein, we isolated 138 bacterial colonies from the fresh bile specimens of four cholecystitis patients using a culturome approach and genomically characterized 35 non-redundant strains using whole-genome shotgun sequencing. The bile bacterial isolates spanned 3 classes, 6 orders, 10 families, and 14 genera, of which the members of Enterococcus, Escherichia-Shigella, Lysinibacillus, and Enterobacter frequently appeared. Genomic analysis identified three species, including Providencia sp. D135, Psychrobacter sp. D093, and Vibrio sp. D074, which are not represented in existing reference genome databases. Based on the genome data, the functional capacity between bile and gut isolates was compared. The bile strains encoded 5,488 KEGG orthologs, of which $4.9 \%$ were specific to the gut strains, including the enzymes involved in biofilm formation, two-component systems, and quorum-sensing pathways. A total of 472 antibiotic resistance genes (ARGs) were identified from the bile genomes including multidrug resistance proteins (42.6\%), fluoroquinolone resistance proteins (12.3\%), aminoglycoside resistance proteins (9.1\%), and $\beta$-lactamase (7.2\%). Moreover, in vitro experiments showed that some bile bacteria have the capabilities for bile salt deconjugation or biotransformation (of primary bile acids into secondary bile acids). Although the physiological or pathological significance of these bacteria needs further exploration, our works expanded knowledge about the genome, diversity, and function of human bile bacteria.

Keywords: bile bacteria, genomic characterization, antibiotic resistance genes, virulence factors, culturome, cholecystitis patients 


\section{INTRODUCTION}

Cholecystitis leads to gallbladder inflammation or even perforation, tissue death, gangrene, fibrosis, and shrinking of the gallbladder, which is a hospitalized disease with increasing medical and financial burden (Wadhwa et al., 2017; Wang et al., 2021). The cardinal symptoms were fever, pain, and nausea with increasing levels of clinical indicators, such as serum transaminases, alkaline phosphatase, and total bilirubin (Chisholm et al., 2019). Laparoscopic cholecystectomy was commonly applied for treating this disease (Loozen et al., 2018).

Although the pathogenicity in cholecystitis still needs further exploration, bacteria, as one of the important risk factors, were closely correlated with its poor operative outcomes (Liu et al., 2015; Yun and Seo, 2018). Moreover, positive bile culture rates from laparoscopic cholecystectomy patients ranged from 25.1 to $60.3 \%$, suggesting the presence of bacteria in the cholecystitis patients' bile (Nitzan et al., 2017; Yun and Seo, 2018). Due to the limitation of culture conditions, most bile bacterial isolates from clinical samples are aerobic bacteria especially Gram stain-negative Enterobacteriaceae, such as Escherichia coli, Klebsiella spp., Enterobacter spp., and less frequently anaerobic bacteria, including Bacteroides and Clostridium spp., as well as microaerophilic Helicobacter pylori (Nitzan et al., 2017; Cen et al., 2018). For culture-independent methods, denaturing gradient gel electrophoresis (DGGE) was applied for the investigation of microbiota composition (Liu et al., 2015); however, low throughput and complex operation were its limitations. With the advent of high-throughput sequencing platforms and bioinformatic analysis pipelines, metagenome sequencing analysis including $16 \mathrm{~S}$ rRNA amplicon sequencing and whole genomic shotgun sequencing have revealed the abundant diversity composition of the bile microbiome ( $\mathrm{Wu}$ et al., 2013; Liu et al., 2015). Meanwhile, owing to nearly $80 \%$ of the human intestinal bacteria were unculturable (Tajeddin et al., 2016), with the advancement in bacterial culturomics technology (Tidjani Alou et al., 2020), it is reasonable to infer that abundant bile bacteria are waiting to be isolated.

Proper identification method of the isolated bacteria was a highly essential step in the culture-dependent workflow. The traditional polyphasic taxonomic strategy aims to generate phenotypic and phylogenetic information of a bacterium, such as its shape, color, size, staining properties, host range, pathogenicity, indole test, gelatin liquefaction test, motility test, and assimilation of carbon sources (Das et al., 2014). In addition to biochemical identification, flow cytometry and MALDI-TOF mass spectrometry can also be used for rapid identification for cultured isolates (Das et al., 2014; Jang and Kim, 2018). Despite each approach having its own advantages and limitations, such as higher cost or rough results of flow cytometer and equipment popularity of MALDI-TOF mass spectrum hindered their application. Considerable advancement in culturable bacterial identification and taxonomy methods using genotypic and phylogenetic information has taken place such as chemotaxonomy, numerical taxonomy, and DNA-DNA hybridization, housekeeping gene amplification, and sequencing (16S rRNA gene, gyrB, rpoABCD, etc.) and whole-genome sequencing (Das et al., 2014). As the primary gene target for bacterial identification and comprehensive consideration of cost performance, the 16S rRNA gene was often used for preliminary screening. Then whole-genome sequencing was used for in-depth exploration of functional genes. In recent years, cultivation-dependent microbiota studies of the human digestive tract (Browne et al., 2016; Lagier et al., 2016), respiratory tract (Fonkou et al., 2018), genitourinary tract (Thomas-White et al., 2018), oral cavity (Fonkou et al., 2018; Martellacci et al., 2020), and even in other model animals (Lagkouvardos et al., 2016; Liu et al., 2020) mostly adopted this strategy and had isolated hundreds of previously unknown bacteria inhabiting the human body. In addition, live bacteria could facilitate the follow-up mechanism researches regarding their impact on physiological and pathological functions, such as the bile salt (salt ion form of bile acid) metabolism test. Bile acids are cholesterol-derived natural surfactants and are regarded as important digestive hormones that are produced in the liver and secreted into the duodenum via the gallbladder to regulate numerous physiological processes in the host (Harris et al., 2018). They are known to affect lipid digestion, antibacterial defense, glucose metabolism, host metabolism, cancer progression, and innate and adaptive immunity (Shapiro et al., 2018; Hang et al., 2019; Song et al., 2020). The biotransformation of bile acids (BAs) by intestinal bacteria may have an important role in cholesterol gallstone formation and colon carcinogenesis via TGR and FXR (farnesoidX receptor) receptors (Salvioli et al., 1982; Funabashi et al., 2020). So far, bacteria are mainly involved in two categories of bile acid metabolism. Bile salt hydrolase (BSH) with deconjugation activity hydrolyzes the amide bond that links the bile acid side chain to glycine or taurine and takes part in the transformation process from conjugated bile acid to unconjugated bile acid (Ridlon et al., 2006). Besides, the dehydroxylase activity participates in the transformation process from primary bile acid to secondary bile acid (White et al., 1980). These metabolic activities have mostly been found in intestinal bacteria (Kuipers et al., 2020). However, the ability of bile acid biotransformation by bile bacteria has not been reported to date.

Altogether, the objectives of the study are to (1) cultivate and isolate a large number of bile bacteria of cholecystitis patients and whole-genome sequence parts of isolates, (2) conduct comparative analyses between our isolates and gut isolates in public databases, which revealed different pathways in our isolates, and (3) evaluate the bile salt metabolic capacity of biliary bacteria in vitro. This work provided new insights into the bile bacterial communities based on genomes and the biotransformation potentiality of human bile bacteria.

\section{MATERIALS AND METHODS}

\section{Sample Collection}

Informed consents were obtained from all subjects, and this study was approved by the Medical Ethics Committee of Hunan Provincial People's Hospital. The study was conducted according to the principles of the Declaration of Helsinki of 1964 and later versions (2013) and was approved by the Medical 
Ethics Committee of the Hunan Provincial People's Hospital (Ethical approval number: 2020-54). All the experiments were performed in accordance with relevant guidelines and regulations. The bile samples collected from four postoperative (laparoscopic cholecystectomy) acute cholecystitis patients in Hunan Provincial People's Hospital were kept in an anaerobic gas bag (Mitsubishi, Japan), respectively, and rapidly transferred to the laboratory for cultivation. To address the potential contamination that might have occurred before and after the samples reached our laboratory, sterile physiological saline control samples without bile were applied in the study.

\section{Cultivation and Identification of Bile Species}

All bile samples were centrifuged at 3,000 $\times g$ for $5 \mathrm{~min}$ and carefully removed most of the supernatant of the bile sample but left around $200 \mu \mathrm{l}$ of supernatant on the bottom to resuspend the pellet to keep bacteria with the highest density. These bacteria were then spread on different solid media for culture. [BBLBrain Heart Infusion Agar (Becton Dickinson, United States), Bifidobacterium Medium (Becton Dickinson, United States), Lactose Bile (Becton Dickinson, United States), Blood Agar Base Infusion Agar (Becton Dickinson, United States), Columbia Blood Agar Base (Becton Dickinson, United States), BBE Agar (Becton Dickinson, United States), and Gifu Anaerobic Medium (Beijing Land Bridge Technology Co., Ltd, China)] under aerobic and anaerobic conditions at $37^{\circ} \mathrm{C}$. Cultivation and identification methods of bile species mainly referred to two studies (Browne et al., 2016; Lagier et al., 2016). Briefly, colonies were picked up and transferred on a fresh medium for the purification of bacterial colony. Isolated colonies were transferred into an 8-ml liquid medium for enrichment culture and were identified using PCR amplification of the 16S rRNA gene (primers: 7F 5' -AGAGTTTGATYMTGGCTCAG-3'; 1510R 5'-ACGGYTACCTTGTTACGACTT-3') (Browne et al., 2016). The PCR products were applied on Sanger sequencing at ABI 3730XL platform (Applied Biosystems, United States) (Browne et al., 2016). Each 16S rRNA sequence was blasted against the rRNA/ITS databases of the National Center of Biotechnology Information (NCBI) for bacterial strain identification. The identified aerobic strains were preserved in 30\% glycerol and anaerobic strains in $30 \%$ glycerol and $0.1 \%$ cysteine at a $-80^{\circ} \mathrm{C}$ freezer.

\section{Whole-Genome Shotgun Sequencing, Assembly, and Annotation}

The DNA of bacterial isolates was extracted using the Qiagen DNA extraction kit (Qiagen, Germany) according to the protocols of the manufacturer. The DNA concentration and purity were determined by NanoDrop2000. DNA quality was examined with a $1 \%$ agarose gel electrophoresis. Bacterial DNA was fragmented to an average size of $\sim 300$ bp using Covaris M220 (Gene Company Limited, China). Paired-end libraries were prepared by using a TruSeq DNA sample prep kit (Illumina, United States). Adapters containing the full complement of sequencing primer hybridization sites were ligated to blunt-end fragments. Paired-end whole-genome shotgun sequencing was performed on the Illumina HiSeq platform. High-quality reads were extracted based on the FASTQ (Chen et al., 2018), with default parameters. High-quality reads were used for de novo assembly via SPAdes (Bankevich et al., 2012), using different $\mathrm{k}$-mer sizes $(k=21,33,55,77)$. The shortest scaffolds were filtered with a minimum length threshold of $200 \mathrm{bp}$. Gene identification was performed from the assembled genome using Prodigal (Hyatt et al., 2010), and the other genomic contents (e.g., rRNA and tRNA sequences) were annotated using the Prokka (Seemann, 2014) pipeline. Protein-coding genes were further annotated to the KEGG (Kyoto Encyclopedia of Genes and Genomes, downloaded on February 2020) (Kanehisa et al., 2017) databases using BLASTP (identity threshold of $35 \%$, covering $>70 \%$ of the gene length).

\section{IDENTIFICATION OF ANTIBIOTIC RESISTANCE GENES AND VIRULENCE FACTORS}

Identification and characterization of antibiotic resistance genes (ARGs) and virulence factors of pathogens are crucial in understanding bacterial pathogenesis and their interactions with the host, and in the development of novel drugs, vaccines, and molecular diagnostic tools. Furthermore, detecting virulence or resistance markers improved outbreak monitoring and therapeutic management. Currently, the use of the nextgeneration sequencing platforms has allowed great progress in this field (Bakour et al., 2016). ARGs were identified based on ABRicate. ${ }^{1}$ ABRicate searched on the databases, including NCBI Bacterial Antimicrobial Resistance Reference Gene Database, CARD (Jia et al., 2017), ARG-ANNOT (Gupta et al., 2014), and ResFinder (Zankari et al., 2012) for predicting ARGs. The virulence factors of the genome were identified based on the VFDB database (Chen et al., 2016).

\section{Comparison Genome Analyses}

Average nucleotide identity (ANI) between two genomes was calculated using the fastANI algorithm (Jain et al., 2018). An ANI threshold of $95 \%$ was used for species delineation for prokaryotic genomes (Jain et al., 2018). The phylogenetic tree of bacterial species was generated using PhyloPhlAn2 (Segata et al., 2013) and visualized using iTOL (Letunic and Bork, 2019).

\section{In vitro Bile Acid Transformations by Bile Bacteria}

Bile acids were dissolved in DMSO for preparation of a highly concentrated stock solution. Then, the autoclaved GAM liquid medium was applied to dilute the stock solution (volume ratio of DMSO in a working solution $<1 \%$ ). The bile acid experimental group [containing $200 \mathrm{ng} / \mathrm{ml}$ of final concentration: TCA (taurocholic acid), CA (cholic acid), or GCA (glycocholic acid)] and the control group (without bile acid) were inoculated

\footnotetext{
${ }^{1}$ https://github.com/tseemann/abricate
} 
with isolated bile bacteria in $200 \mu \mathrm{l}$ of liquid media reaction system at $37^{\circ} \mathrm{C}$. A control without bile acid (but with the DMSO vehicle) and a sterile control with an equal volume of bile acid $(200 \mathrm{ng} / \mathrm{ml})$ were prepared. Every reaction was prepared in parallel. This experiment was done in triplicates. Bile acids of internal standards were prepared at $200 \mathrm{ng} / \mathrm{ml}$. After $12 \mathrm{~h}$, the cultures were centrifuged $(10,000 \times g, 5 \mathrm{~min})$, and the supernatants were collected. Then, the double volume of acetonitrile was added, and the aqueous phase was collected after centrifuging at 20,000 $\times g$ for $10 \mathrm{~min}$. The detection was achieved using a Phenomenex Kinetex Polar C18 ODS $(2.1 \times 100 \mathrm{~mm}$, $2.6 \mu \mathrm{m}$ ) analytical column. The mobile phase consisted of $0.1 \%$ ammonium hydroxide water (A) and acetonitrile (B) at a flow rate of $0.3 \mathrm{ml} / \mathrm{min}$, and the following gradient condition was used: $0-1 \min 65 \% \mathrm{~A} ; 1-4 \min 65-60 \% \mathrm{~A} ; 4-5.5 \min 60-35 \%$ A; 5.5-6.5 min 35-20\% A, 6.5-7.0 20-10\% A, 7.0-8.0 10\% A, 8.0-8.5 10-65\% A). An Applied Biosystems AB Sciex Qtrap5500 Mass Spectrometer (MS/MS) equipped with an electron spray ionization (ESI) source was used to analyze target metabolites at negative ionization mode (Scherer et al., 2009). The optimized ion spray voltage and drying gas temperature were set at $4,500 \mathrm{~V}$ and $550^{\circ} \mathrm{C}$, respectively. The curtain gas (CUR) flow was $35 \mathrm{~L} / \mathrm{min}$; gas 1 and gas2 (nitrogen) were set at 45 and $55 \mathrm{psi}$, respectively, and the retention time was $70 \mathrm{~ms}$. Quantification assays were performed using multiple reaction monitoring.

\section{Statistical Analyses}

The value of metabolite output is equal to the output of the experimental group minus the average output of the control group. All statistical analyses were performed based on the $\mathrm{R}$ platform. Histograms were performed using GraphPad Prism 7 software (GraphPad Software) and were indicated using the mean with the standard deviation.

\section{RESULTS}

\section{Cultivation and Genome Sequencing of Bacteria in Bile Specimens}

To explore the bile bacterial community, the fresh bile specimens collected from cholecystitis patients were cultivated using seven different media under both aerobic and anaerobic conditions. In total, we obtained 138 bacterial colonies and performed full-length 16S rRNA gene sequencing to enable taxonomic assignment. These isolates included 70 (50.7\%) members of Firmicutes, 65 (47.1\%) members of Proteobacteria, and 3 (2.2\%) members of Actinobacteria; meanwhile, they expanded 3 classes, 6 orders, 10 families, and 14 genera (Supplementary Table 1). At the genus level, Enterococcus (31.9\%), Escherichia-Shigella (22.9\%), Lysinibacillus (9.4\%), and Enterobacter (8\%) were the most frequent members in the biliary community, while the remaining 10 genera represented 28.2\% (39/138) of the cultivated isolates (Figure 1A). Proteus mirabilis (24 strains) and Enterococcus faecalis (17 strains) were the most abundant species, which may be due to the property of facultative anaerobes (Portela et al., 2014; Jamil et al., 2021). Enterobacteriaceae had the largest isolated strains (28 strains) under aerobic conditions
(Supplementary Table 1). In addition, 63 strains were obtained under anaerobic conditions (63/138), and most of them were also facultative anaerobes, such as the Enterococcus genus.

We clustered the isolates into 35 operational taxonomic units (OTUs) based on 99\% nucleotide similarity of their 16S rRNA gene sequences and performed whole-genome sequencing for the representative isolate for each cluster. After de novo assembly of the sequencing reads, we obtained 35 high-quality draft genomes that exceeded 95\% genomic completeness for each strain (Figure 1B). A phylogenetic tree of the draft genomes was shown in Figure 1C. The genome sizes of these biliary bacterial isolates were on average $4.0 \mathrm{Mbp}$, ranging from 1.9 to $7.1 \mathrm{Mbp}$ (Supplementary Table 2), and the G + C contents of these genomes ranged from 37.4 to $63.3 \%$ (on average, $47.4 \%$ ). The majority $(32 / 35)$ of the biliary genomes could be assigned into known species, showing $>95 \%$ ANI to at least one sequenced genome in the NCBI database, whereas the other 3 biliary isolates were "novel species" as they previously had no available whole-genome information (Supplementary Table 2). Moreover, despite the 16S rRNA gene sequences of these biliary strains seemed diverse, 26 genomes were further grouped into 8 specieslevel genome bins based on $95 \%$ ANI.

\section{Characteristics of the Novel Species}

We analyzed the genomes of three novel species to investigate the distinctive features of those biliary bacterial species. Biliary strain Providencia sp. D135 consisted of 27 contigs with a total length of $4.45 \mathrm{Mbp}$ (N50 length: $315 \mathrm{kbp}$ ). This strain was moderate homology with the genome of Providencia sp. WCHPr000369, a clinical strain that was isolated from the human rectum (proposed name: Providencia huaxiensis) (Hu et al., 2019), with 92.3\% ANI (Figure 2A). Biliary strain Psychrobacter sp. D093 consisted of 99 contigs with a total length of $2.69 \mathrm{Mbp}$ and showed $82.9 \%$ ANI with the genome of Psychrobacter cryohalolentis K5 (Figure 2B). Biliary strain Vibrio sp. D074 consisted of 87 contigs with a total length of $3.93 \mathrm{Mbp}$ and showed $79.6 \%$ ANI with the genome of Vibrio anguillarum VIB43 (Figure 2C).

\section{Comparison of Bile and Gut Isolates}

To investigate the specificity of bile bacterial species, we compared our bile isolates with 1,520 bacterial strains cultivated from the gastrointestinal tract of healthy adults (Zou et al., 2019). Based on wholegenome pairwise ANI, 13 strains were specifically detected in bile samples. These bile-specific genomes were mainly distributed in Firmicutes, including Lactobacillus murinus, Enterococcus casseliflavus, Paenibacillus lautus, Staphylococcus pettenkoferi, Lysinibacillus sp. D060, and Solibacillus silvestris, and several Proteobacteria clades, including Providencia sp. D135, Morganella morganii, Vibrio sp. D074, and Psychrobacter sp. D093 (Supplementary Figure 1).

The functional roles of the members of bile and gut strains were compared based on their profiles of the KEGG orthologs (KOs). The bile strains encoded 5,488 KOs, of which $95.1 \%$ 


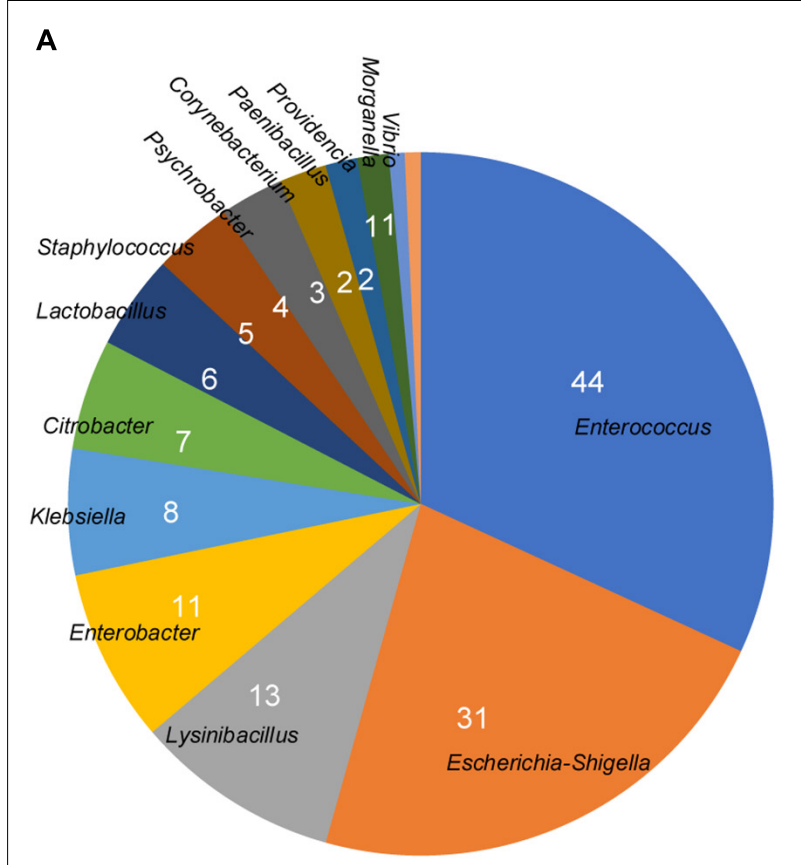

\section{B}

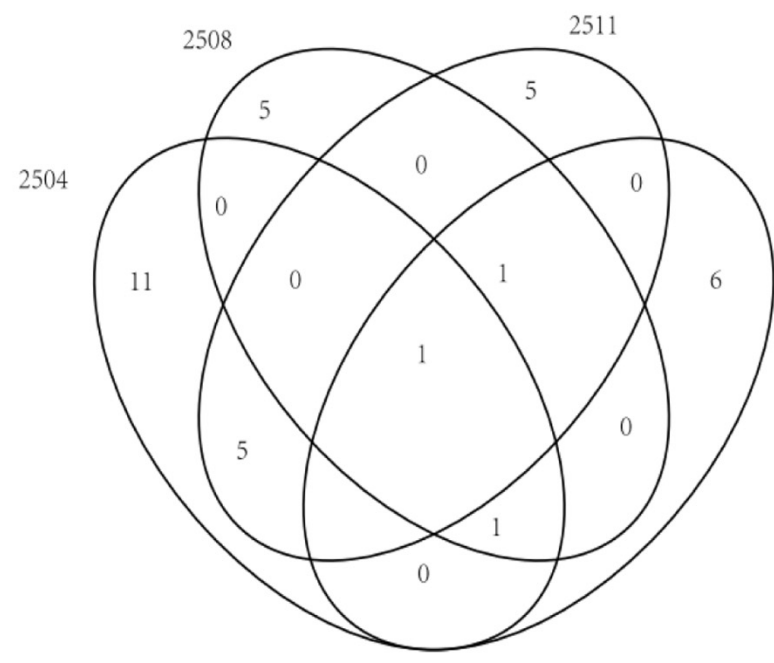

2514

C

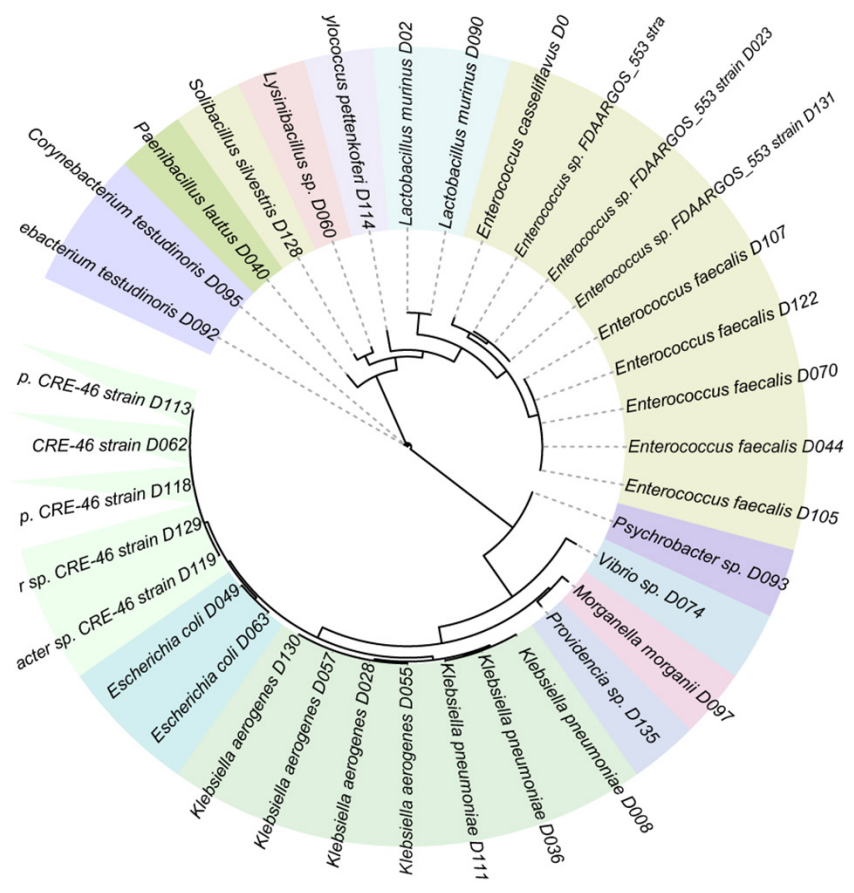

\begin{tabular}{l} 
Genus: \\
\hline$\square$ Citrobacter \\
$\square$ Corynebacterium \\
$\square$ Escherichia \\
$\square$ Klebsiella \\
$\square$ Lactobacillus \\
$\square$ Lysinibacillus \\
$\square$ Paenibacillus \\
$\square$ Providencia \\
$\square$ Psychrobacter \\
$\square$ Solibacillus \\
$\square$ Staphylococcus \\
$\square$ Vibrio \\
$\square$ Xenorhabdus \\
Tree scale: 1 म
\end{tabular}

FIGURE 1 | Overview of the cultivated bacteria from bile specimens. (A) Pie plot shows the composition of 138 bacterial colonies. (B) Venn diagram of the distribution of isolates in the four bile samples. (C) Phylogenetic tree of 35 whole-genome sequenced isolates. Color represents the genus assignment of the isolates.

were also encoded by the gut strains (Figure 3A). The bilespecific KOs were involved in pathways including biofilm formation, two-component systems, quorum sensing, and $\mathrm{ABC}$ transporters (Figure 3B). We also undertook an enrichment analysis and identified 3,740 statistically enriched KOs and 695 reduced KOs in bile bacteria (Benjamini-Hochberg-adjusted $p<0.05$, Fisher's exact test). The bile-enriched KOs had frequently performed some key functions, including $A B C$ transporters, two-component system, quorum sensing, purine metabolism, biofilm formation, oxidative phosphorylation, pyruvate metabolism, and phosphotransferase system, while the bile-reduced KOs were involved in functions such as 


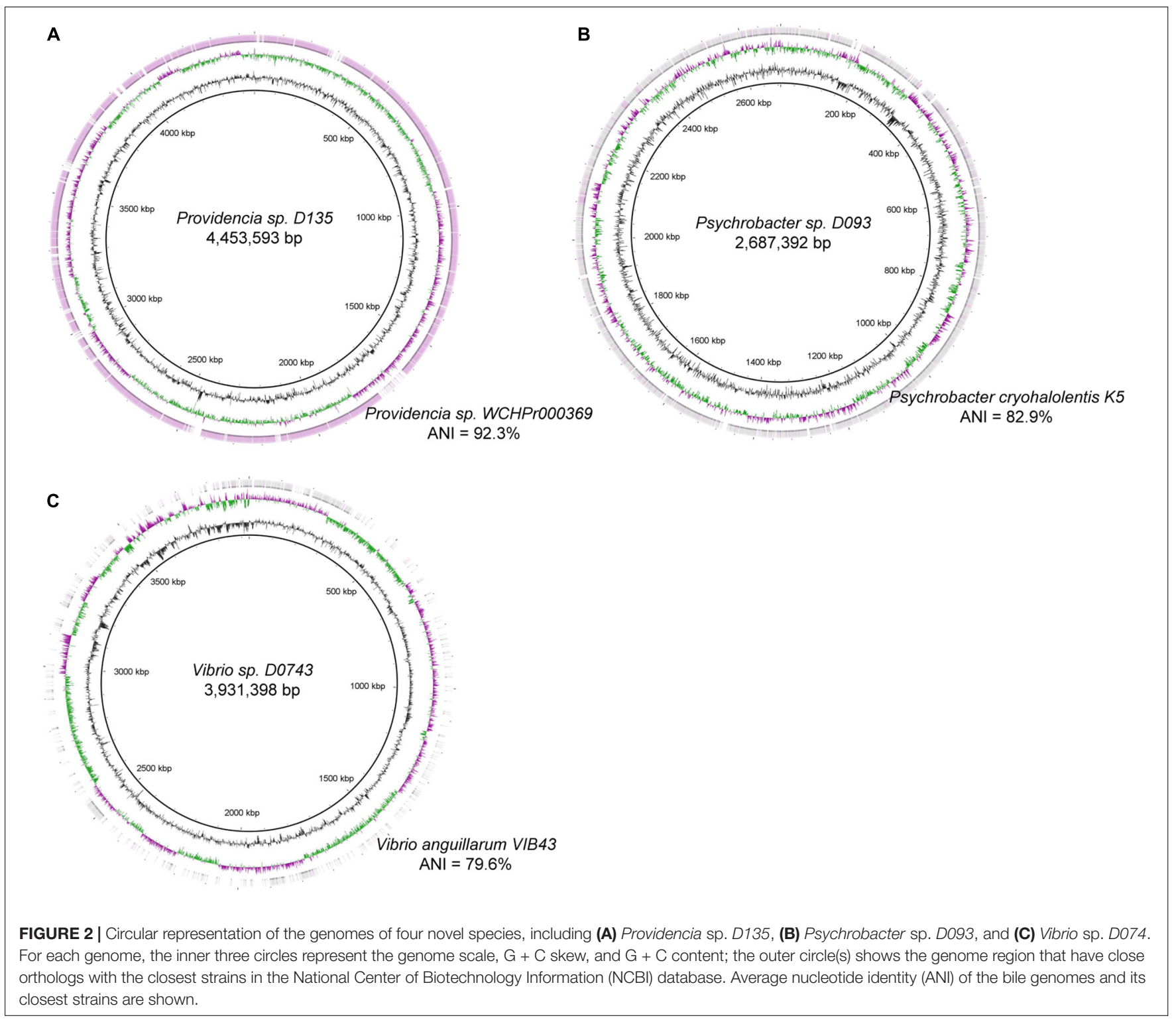

the ribosome, two-component system, amino sugar and nucleotide sugar metabolism, and starch and sucrose metabolism (Figures 3C,D).

\section{Antibiotic Resistance Genes, Virulence Factors, and Bile Acid Metabolism Genes}

Next, we examined the existence of ARGs and virulence factors in the genomes of bile species, aiding to extend their potential clinical pathologic features. A total of 472 genes from 27 genomes were identified as ARGs based on annotations in the available antibiotic resistance databases (Supplementary Table 3). Most of the ARGs were related to multidrug resistance $(\mathrm{MDR})$ (42.6\%), fluoroquinolone resistance $(12.3 \%)$, aminoglycoside resistance $(9.1 \%)$, and $\beta$-lactamase $(7.2 \%)$. The remaining genes were involved in resistance against various types of antibiotics such as tetracycline (4.4\%), glycopeptide
(4\%), peptide (4\%), and trimethoprim (4\%). At the species level, Escherichia coli, Citrobacter sp. CRE-46, Klebsiella aerogenes, and Klebsiella pneumoniae encoded a higher number of ARGs than other bile species, especially that their ARGs were enriched in types including MDR, fluoroquinolone resistance, and $\beta$ lactamase (Figure 4A). Enterococcus faecalis frequently encoded the genes involving trimethoprim, macrolide, and tetracycline resistances, while Enterococcus casseliflavus and Enterococcus sp. FDAARGOS_553 encoded the genes of glycopeptide resistance. In addition, we identified 354 genes involving virulence factors from 20 bile strains (Supplementary Table 4). The virulence factors were mainly distributed in species including Escherichia coli, Enterococcus faecalis, Klebsiella pneumoniae, Citrobacter sp. CRE-46, and Klebsiella aerogenes (Figure 4B). In addition, we identified a total of 12 genes encoding the $\mathrm{BSH}$ and 319 genes involved in the transformation of primary bile acids to secondary bile acids (i.e., the multiple steps 
A

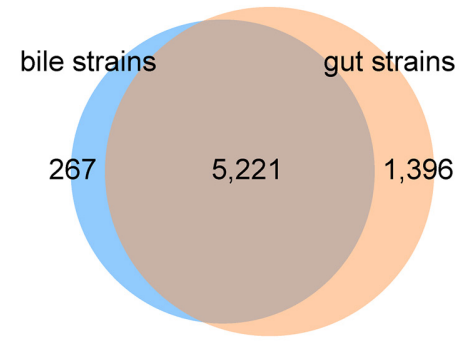

C

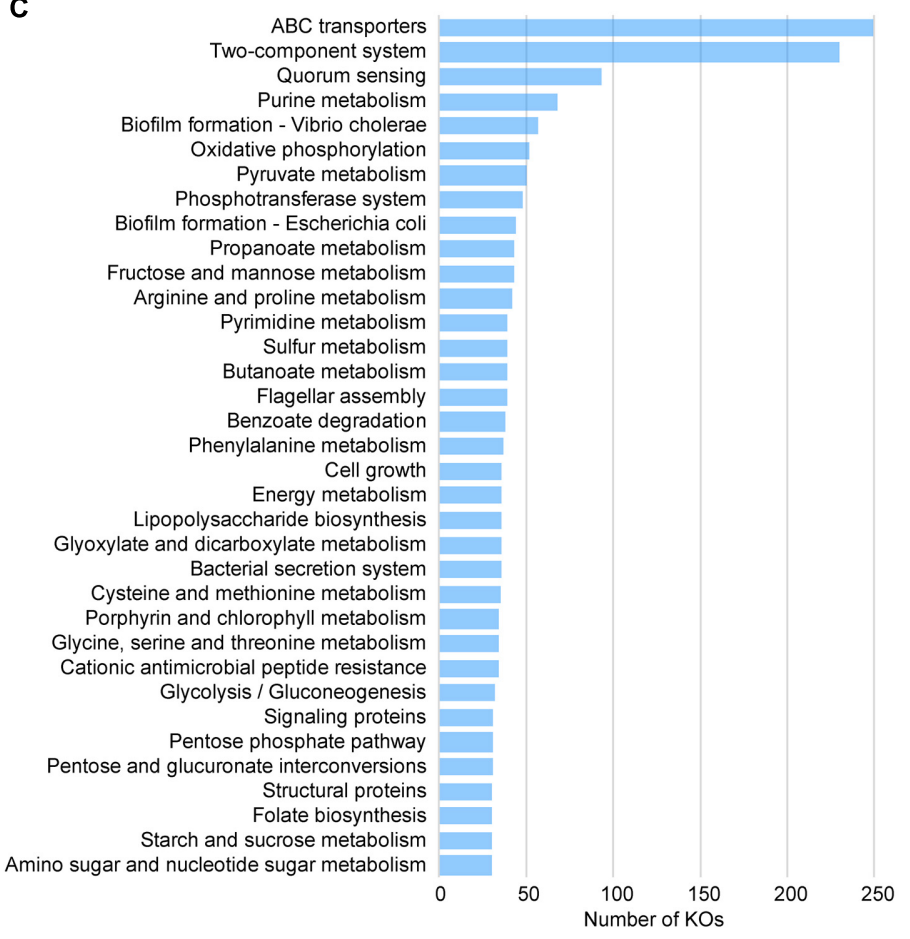

B Biofilm formation - Vibrio cholerae Two-component system Quorum sensing
ABC transporters Arabinogalactan biosynthesis - Mycobacterium Oxidative phosphorylation

Vibrio cholerae infection Lipoarabinomannan (LAM) biosynthesis Styrene degradation
Cysteine and methionine metabolism Steroid degradation Benzoate degradation Biosynthesis of enediyne antibiotics Biosynthesis of unsaturated fatty acids Arginine and proline metabolism Huntington disease Bacterial secretion system

$$
10 \quad 20
$$

D

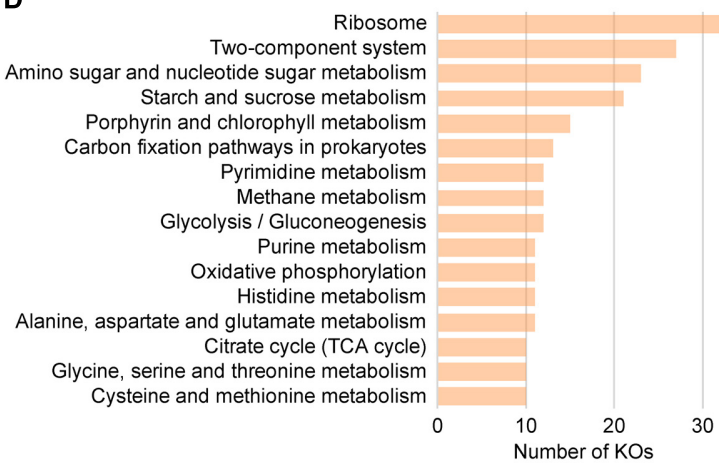

FIGURE 3 | Comparison of the functions of bile and gut bacterial strains. (A) Venn diagram shows the comparison of KEGG orthologs (KOs) between bile and gut strains. (B-D) Distribution of KEGG level C pathways of the bile-specific KOs (B), the bile-enriched KOs (C), and the gut-enriched KOs (D).

$7 \alpha / \beta$-dehydroxylation) (Supplementary Table 5). These genes spanned all 35 genome-sequenced strains, suggesting that the bile bacteria have widely participated in bile acid metabolism. At the species level, Lysinibacillus sp. D060, Solibacillus silvestris, Paenibacillus lautus, and the members of Klebsiella (i.e., $K$. aerogenes and $K$. pneumoniae) had the largest number of bile acid metabolism genes.

\section{Evaluation of Bile Salt Metabolism Potentiality of Bile Bacteria}

We evaluated the potential BSH and dehydroxylase activities in 24 strains, which were selected by phylogenetic tree based on $16 \mathrm{~S}$ rRNA gene amplicon sequence by measuring the changed concentration of free bile acids or secondary bile acids in the supernatant of cultures. Paenibacillus sp. H203 (Ps) and Lysinibacillus xylanilyticus Gute33 (Lx) were detected with strong bile salt deconjugation capabilities. Cholic acid formed with GCA substrate $[586.1 \pm 135 \mathrm{ng} / \mathrm{ml}(\mathrm{Ps}), 445.4 \pm 14.1 \mathrm{ng} / \mathrm{ml}(\mathrm{Lx})]$ and $1,500.6 \pm 42 \mathrm{ng} / \mathrm{ml}(\mathrm{Ps}), 1,525.4 \pm 49 \mathrm{ng} / \mathrm{ml}(\mathrm{Lx})$ cholic acid formed with TCA substrate (Figure 5A). Lysinibacillus sp. MJJ11 and Corynebacterium testudinoris DSM 44614 strain showed the stronger capabilities of forming secondary bile acid, which converted CA to DCA (Figure 5B).

\section{DISCUSSION}

The bile bacteria, including the Vibrio genus especially Vibrio cholerae (Asnis et al., 1996), Escherichia coli, Klebsiella spp., Enterococcus (Yun and Seo, 2018), Staphylococcus, Corynebacterium (Backert et al., 2018), and Lactobacillus salivarius, have been studied extensively (Woo et al., 2002). Tajeddin et al. (2016) found that enterobacteria were the dominant biliary bacteria based on cultivate-dependent methods. As an alternative animal model of humans, most of the bile and gall bladder bacteria of healthy pigs were Streptococcus alactolyticus, Staphylococcus epidermidis, and Corynebacterium testudinoris, which suggested that healthy host bile may also 

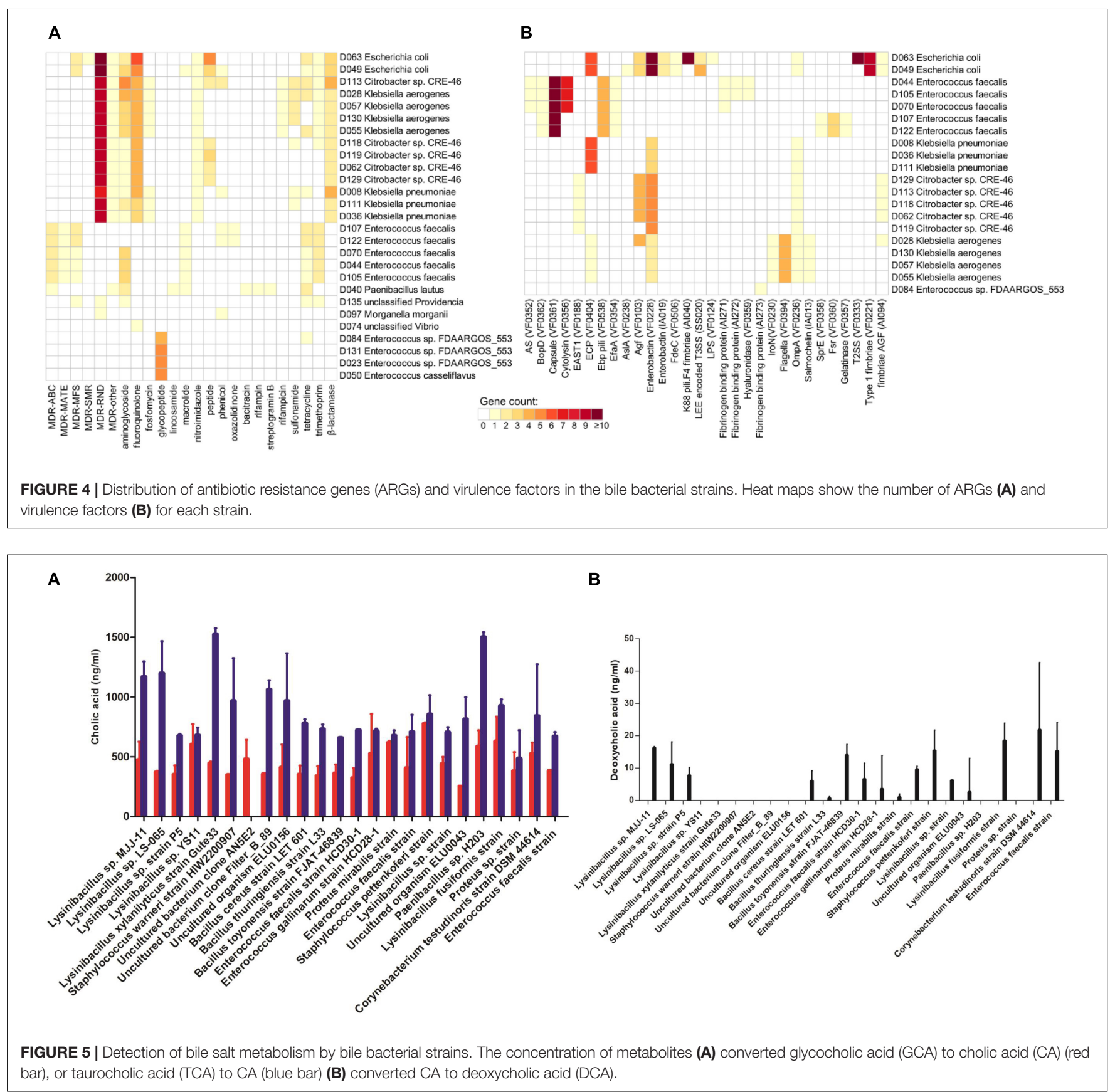

contain bacteria (Jimenez et al., 2014). In our previous research and the work of others, the bile pathogenic bacteria were considered a predisposing factor in gallbladder disorders (Liu et al., 2015; Shen et al., 2015; Tajeddin et al., 2016). Furthermore, metagenomic results indicated that many potential bile bacteria still cannot be isolated and cultivated (Liu et al., 2015). With more cultivated and detected conditions termed "culturomics" emerging (Lagier et al., 2016), many previously unavailable "dark matter bacteria" from bile would be isolated.

In this study, we characterized the isolated bacterial genome from the fresh bile specimens from four cholecystitis patients and evaluated its bile acid biotransformation ability in vitro. Our results showed that the number of cultivable bile bacteria exceeds expectations, spanning 138 bacterial isolates that were distributed in 14 genera. In particular, three bacterial species (i.e., Providencia sp. D135, Psychrobacter sp. D093, and Vibrio sp. D074) were not represented in existing reference genome databases. Enterobacteriaceae had the most isolated strains (28 strains) under aerobic conditions, in agreement with previous studies (Tajeddin et al., 2016). Although there was little research on bile anaerobes, we obtained 63 strains under anaerobic conditions, however, most of them were facultative anaerobes. 
The possible reasons were the small sample size $(n=4)$ and the nutrient composition of the medium, which was not specifically optimized or not rich enough for bile bacteria. For instance, Lagier et al. (2016) reported that liquid media precultivation operation can effectively promote the diversity of isolated bacteria. In addition, the centrifugal operation may lose some lighter bacteria; therefore, after centrifugation, the supernatant can be aseptically filtered (Spin$\mathrm{X}$ Centrifuge Tube Filter, Corning Inc.) and concentrated, and the concentrated part will be used for cultivation to obtain more bacteria. The isolated bacterial genomes could extend the databases, which are the basis for metagenomics analysis (Lagier et al., 2016).

Whole-genome sequencing was performed on 35 bacterial species and compared with gut isolates. Our results showed that many pathways, such as biofilm formation, ABC transporters, and quorum sensing, were enriched in bile bacteria, which may contribute to communicate with each other and resist the antibacterial high concentration of bile salts (Davidson et al., 2008; Moons et al., 2009; Rutherford and Bassler, 2012). Bile salts promoted the biofilm formation of bile bacteria, and the biofilms of bacteria-laden gallstones influenced the illness severity (Stewart et al., 2007). Especially, the biofilm formation ability seems to be a necessary condition for bile resistance and polymicrobial infection in this organ (Tajeddin et al., 2016; Zheng et al., 2017). Therefore, this high abundance of functional pathways of bile bacteria, such as biofilm formation, was related to its living environment and physiological characteristics.

Enterobacteriaceae had the largest isolated strains (28 strains) under aerobic conditions in this study which was consistent with the previous studies (Yun and Seo, 2018) and had been identified to be closely related to cholecystitis in our previous research (Liu et al., 2015). ARGs and virulence factors analyses of the bacterial isolates further revealed that important Gramnegative opportunistic pathogen of the Enterobacteriaceae, such as Escherichia coli, Citrobacter sp. CRE-46, Klebsiella aerogenes, and Klebsiella pneumoniae, encoded the higher number of ARGs and the virulence factors than other bile species. This result was inconsistent with those of previous studies that less than $5 \%$ resistance was observed in bile isolated bacteria against carbapenem, beta-lactam antibiotics, glycopeptide antibiotics, and linezolid via the antibiotic susceptibility tests (Yun and Seo, 2018). This difference may be caused by different test methods and their sensitivity. Therefore, the combination of the bioinformatics and the verification of conventional drugsusceptibility testing method on the pivotal isolates in the next step may have positive significance in the quicker selection of the most suitable antibiotics for clinical applications.

In humans, the major bile salts included taurocholic acid (TCA) and glycocholic acid (GCA) derived from cholic acid (CA), while taurochenodeoxycholic acid (TCDCA) and glycochenodeoxycholic acid (GCDCA) derived from chenodeoxycholic acid (CDCA). The salts of their 7-alphadehydroxylated derivatives, deoxycholic acid (DCA), and lithocholic acid (LCA), are also found, with derivatives of cholic, chenodeoxycholic, and deoxycholic acids accounting for over $90 \%$ of human biliary bile acids (Hofmann, 1999). Bile acids are closely related to human health and can be metabolized by gut bacteria, which generated free bile acid by deconjugation and generated secondary bile acids by $7 \alpha$-and $7 \beta$-dehydroxylation, such as deoxycholic acid and lithocholic acid (Ridlon et al., 2016; Wang and Jia, 2016; Marion et al., 2019; Song et al., 2019; Funabashi et al., 2020). Secondary bile acids, especially DCA, have been listed as carcinogens and promoters of CRC (Yang and Yu, 2018). High-fat diet-induced high levels of DCA in the intestine are also considered an important risk factor for CRC. Herein, based on the phylogenetic tree of isolates, we selected 24 bile bacterial strains and detected their bile salt deconjugation and transformation abilities. Lysinibacillus genus had strong bile acid biotransformation ability (Figure 5), which was consistent with previous reports that Paenibacillus and Lysinibacillus widely carried BSH (Song et al., 2019). This ability of bile bacteria may allow them to overcome the bile salt damaging effect. Although this study provided direct evidence in vitro for the bile bacteria involved in the biotransformation of bile acid, however, we are not sure that the bacteria-mediated transformations occur in the gallbladder or not so far. The reason is that the presence of in vivo bile acid transformations performed by certain isolated microorganisms lacks rigor since the same strain may display completely different functional performance between its in vivo and in vitro activity (Marion et al., 2019). For instance, Clostridium hiranonis did not deconjugate tauro-conjugated bile acids in germ-free mice, although its ability to deconjugate taurocholic acid (TCA) was shown in vitro (Narushima et al., 1999). Importantly, our research results provided new insights for screening bile salt metabolizing bacteria, which many studies focused on gut bacteria (Lucas et al., 2021).

Our data helps in understanding the genetic basis of the physiology, biochemical pathways, and evolution of the isolates and provides a preliminary exploration in bile acid transformations by bile bacteria; however, the sample size and culture conditions were relatively small. Therefore, larger-scale bacterial isolation and identification, more in-depth screening isolates with bile acid transformation ability in vivo by metabolite detection method, and verifying the impact of metabolite on gallbladder and even whole-body health are to be investigated in the next step.

\section{DATA AVAILABILITY STATEMENT}

The datasets presented in this study can be found in online repositories. The names of the repository/repositories and accession number(s) can be found below: https://www.ncbi.nlm. nih.gov/bioproject/PRJNA726166, PRJNA726166.

\section{ETHICS STATEMENT}

The studies involving human participants were reviewed and approved by the Medical Ethics Committee of the Hunan Provincial People's Hospital (Ethical approval number: 202054). The patients/participants provided their written informed consent to participate in this study. 


\section{AUTHOR CONTRIBUTIONS}

YM, QY, SL, YX, and XHM contributed to conception and design of the study. SL, SZ, YZ, QL, and AZ organized the formal analysis. GW, RL, MX, TJ, YS, XL, SC, XT, and XD performed methodology. YM, XCM, CW, and YX were responsible for project administration. QY, SL, and SZ wrote the first draft of the manuscript. All authors were involved in preparing the manuscript and contributed to manuscript revision, read, and approved the submitted version.

\section{FUNDING}

YM was supported by the National Natural Science Foundation of China (81930112 and 81573469). QY was supported by the

\section{REFERENCES}

Asnis, D. S., Golub, R., and Bresciani, A. (1996). Vibrio cholerae 01 isolated in the gallbladder of a patient presenting with cholecystitis. Am. J. Gastroenterol. 91, 2241-2242.

Backert, S., Tegtmeyer, N., Oyarzabal, O. A., Osman, D., Rohde, M., Grutzmann, R., et al. (2018). Unusual manifestation of live Staphylococcus saprophyticus, Corynebacterium urinapleomorphum, and Helicobacter pylori in the gallbladder with cholecystitis. Int. J. Mol. Sci. 19:1826. doi: 10.3390/ijms1907 1826

Bakour, S., Sankar, S. A., Rathored, J., Biagini, P., Raoult, D., and Fournier, P. E. (2016). Identification of virulence factors and antibiotic resistance markers using bacterial genomics. Future Microbiol. 11, 455-466. doi: 10.2217/fmb.15. 149

Bankevich, A., Nurk, S., Antipov, D., Gurevich, A. A., Dvorkin, M., Kulikov, A. S., et al. (2012). SPAdes: a new genome assembly algorithm and its applications to single-cell sequencing. J. Comput. Biol. 19, 455-477. doi: 10.1089/cmb.2012. 0021

Browne, H. P., Forster, S. C., Anonye, B. O., Kumar, N., Neville, B. A., Stares, M. D., et al. (2016). Culturing of 'unculturable' human microbiota reveals novel taxa and extensive sporulation. Nature 533, 543-546. doi: 10.1038/nature1 7645

Cen, L., Pan, J., Zhou, B., Yu, C., Li, Y., Chen, W., et al. (2018). Helicobacter Pylori infection of the gallbladder and the risk of chronic cholecystitis and cholelithiasis: a systematic review and meta-analysis. Helicobacter 23:e12457. doi: 10.1111/hel.12457

Chen, L., Zheng, D., Liu, B., Yang, J., and Jin, Q. (2016). VFDB 2016: hierarchical and refined dataset for big data analysis-10 years on. Nucleic Acids Res. 44, D694-D697. doi: 10.1093/nar/gkv1239

Chen, S., Zhou, Y., Chen, Y., and Gu, J. (2018). fastp: an ultra-fast all-in-one FASTQ preprocessor. Bioinformatics 34, i884-i890. doi: 10.1093/bioinformatics/bt y560

Chisholm, P. R., Patel, A. H., Law, R. J., Schulman, A. R., Bedi, A. O., Kwon, R. S., et al. (2019). Preoperative predictors of choledocholithiasis in patients presenting with acute calculous cholecystitis. Gastrointest. Endosc. 89, 977-983 e972. doi: 10.1016/j.gie.2018.11.017

Das, S., Dash, H. R., Mangwani, N., Chakraborty, J., and Kumari, S. (2014). Understanding molecular identification and polyphasic taxonomic approaches for genetic relatedness and phylogenetic relationships of microorganisms. J. Microbiol. Methods 103, 80-100. doi: 10.1016/j.mimet.2014.05.013

Davidson, A. L., Dassa, E., Orelle, C., and Chen, J. (2008). Structure, function, and evolution of bacterial ATP-binding cassette systems. Microbiol. Mol. Biol. Rev. 72, 317-364. doi: 10.1128/MMBR.00031-07

Fonkou, M. D., Dufour, J. C., Dubourg, G., and Raoult, D. (2018). Repertoire of bacterial species cultured from the human oral cavity and respiratory tract. Future Microbiol. 13, 1611-1624. doi: 10.2217/fmb-2018-0181
National Natural Science Foundation of China (81902037) and the Natural Science Foundation of Liaoning Province, China (20180530086). XHM was supported by the Natural Science Foundation of Hunan Province, China (2019JJ80007) and the Key Scientific Research Project of Hunan Provincial Education Department, China (20A312).

\section{SUPPLEMENTARY MATERIAL}

The Supplementary Material for this article can be found online at: https://www.frontiersin.org/articles/10.3389/fmicb. 2021.739621/full\#supplementary-material

Supplementary Figure 1 | Phylogenetic tree of 35 bile isolates and 1,520 bacterial strains cultivated from the feces. Outer color bars represent the phylum assignment of the isolates.

Funabashi, M., Grove, T. L., Wang, M., Varma, Y., McFadden, M. E., Brown, L. C., et al. (2020). A metabolic pathway for bile acid dehydroxylation by the gut microbiome. Nature 582, 566-570. doi: 10.1038/s41586-020-2 396-4

Gupta, S. K., Padmanabhan, B. R., Diene, S. M., Lopez-Rojas, R., Kempf, M., Landraud, L., et al. (2014). ARG-ANNOT, a new bioinformatic tool to discover antibiotic resistance genes in bacterial genomes. Antimicrob. Agents Chemother. 58, 212-220. doi: 10.1128/AAC.01310-13

Hang, S., Paik, D., Yao, L., Kim, E., Trinath, J., Lu, J., et al. (2019). Bile acid metabolites control TH17 and Treg cell differentiation. Nature 576, 143-148. doi: 10.1038/s41586-019-1785-z

Harris, S. C., Devendran, S., Mendez-Garcia, C., Mythen, S. M., Wright, C. L., Fields, C. J., et al. (2018). Bile acid oxidation by Eggerthella lenta strains C592 and DSM 2243(T). Gut Microbes 9, 523-539. doi: 10.1080/19490976.2018. 1458180

Hofmann, A. F. (1999). The continuing importance of bile acids in liver and intestinal disease. Arch. Intern. Med. 159, 2647-2658. doi: 10.1001/archinte.159. 22.2647

Hu, Y., Feng, Y., Zhang, X., and Zong, Z. (2019). Providencia huaxiensis sp. nov., recovered from a human rectal swab. Int. J. Syst. Evol. Microbiol. 69, 2638-2643. doi: 10.1099/ijsem.0.003502

Hyatt, D., Chen, G. L., Locascio, P. F., Land, M. L., Larimer, F. W., and Hauser, L. J. (2010). Prodigal: prokaryotic gene recognition and translation initiation site identification. BMC Bioinformatics 11:119. doi: 10.1186/1471-2105-1 $1-119$

Jain, C., Rodriguez, R. L., Phillippy, A. M., Konstantinidis, K. T., and Aluru, S. (2018). High throughput ANI analysis of $90 \mathrm{~K}$ prokaryotic genomes reveals clear species boundaries. Nat. Commun. 9:5114. doi: 10.1038/s41467-018-07 641-9

Jamil, R. T., Foris, L. A., and Snowden, J. (2021). Proteus Mirabilis Infections. Treasure Island, FL: StatPearls.

Jang, K. S., and Kim, Y. H. (2018). Rapid and robust MALDI-TOF MS techniques for microbial identification: a brief overview of their diverse applications. J. Microbiol. 56, 209-216. doi: 10.1007/s12275-018-7 457-0

Jia, B., Raphenya, A. R., Alcock, B., Waglechner, N., Guo, P., Tsang, K. K., et al. (2017). CARD 2017: expansion and model-centric curation of the comprehensive antibiotic resistance database. Nucleic Acids Res. 45, D566D573. doi: 10.1093/nar/gkw1004

Jimenez, E., Sanchez, B., Farina, A., Margolles, A., and Rodriguez, J. M. (2014). Characterization of the bile and gall bladder microbiota of healthy pigs. Microbiologyopen 3, 937-949. doi: 10.1002/mbo3.218

Kanehisa, M., Furumichi, M., Tanabe, M., Sato, Y., and Morishima, K. (2017). KEGG: new perspectives on genomes, pathways, diseases and drugs. Nucleic Acids Res. 45, D353-D361. doi: 10.1093/nar/gkw 1092 
Kuipers, F., de Boer, J. F., and Staels, B. (2020). Microbiome modulation of the host adaptive immunity through bile acid modification. Cell Metab. 31, 445-447. doi: 10.1016/j.cmet.2020.02.006

Lagier, J. C., Khelaifia, S., Alou, M. T., Ndongo, S., Dione, N., Hugon, P., et al. (2016). Culture of previously uncultured members of the human gut microbiota by culturomics. Nat. Microbiol. 1:16203. doi: 10.1038/nmicrobiol.201 6.203

Lagkouvardos, I., Pukall, R., Abt, B., Foesel, B. U., Meier-Kolthoff, J. P., Kumar, N., et al. (2016). The mouse intestinal bacterial collection (miBC) provides host-specific insight into cultured diversity and functional potential of the gut microbiota. Nat. Microbiol. 1:16131. doi: 10.1038/nmicrobiol.201 6.131

Letunic, I., and Bork, P. (2019). Interactive Tree Of Life (iTOL) v4: recent updates and new developments. Nucleic Acids Res. 47, W256-W259. doi: 10.1093/nar/ gkz239

Liu, C., Zhou, N., Du, M. X., Sun, Y. T., Wang, K., Wang, Y. J., et al. (2020). The mouse gut microbial Biobank expands the coverage of cultured bacteria. Nat. Commun. 11:79. doi: 10.1038/s41467-019-13836-5

Liu, J., Yan, Q., Luo, F., Shang, D., Wu, D., Zhang, H., et al. (2015). Acute cholecystitis associated with infection of Enterobacteriaceae from gut microbiota. Clin. Microbiol. Infect. 21, 851 e851-e859. doi: 10.1016/j.cmi.2015. 05.017

Loozen, C. S., van Santvoort, H. C., van Duijvendijk, P., Besselink, M. G., Gouma, D. J., Nieuwenhuijzen, G. A., et al. (2018). Laparoscopic cholecystectomy versus percutaneous catheter drainage for acute cholecystitis in high risk patients (CHOCOLATE): multicentre randomised clinical trial. BMJ 363:k3965. doi: 10.1136/bmj.k3965

Lucas, L. N., Barrett, K., Kerby, R. L., Zhang, Q., Cattaneo, L. E., Stevenson, D., et al. (2021). Dominant bacterial phyla from the human gut show widespread ability to transform and conjugate bile acids. mSystems 6:e0080521. doi: 10 . 1128/mSystems.00805-21

Marion, S., Studer, N., Desharnais, L., Menin, L., Escrig, S., Meibom, A., et al. (2019). In vitro and in vivo characterization of clostridium scindens bile acid transformations. Gut Microbes 10, 481-503. doi: 10.1080/19490976.2018. 1549420

Martellacci, L., Quaranta, G., Fancello, G., D’Addona, A., Sanguinetti, M., Patini, R., et al. (2020). Characterizing peri-implant and sub-gingival microbiota through culturomics. first isolation of some species in the oral cavity. a pilot study. Pathogens 9:365. doi: 10.3390/pathogens 905 0365

Moons, P., Michiels, C. W., and Aertsen, A. (2009). Bacterial interactions in biofilms. Crit. Rev. Microbiol. 35, 157-168. doi: 10.1080/1040841090280 9431

Narushima, S., Itoh, K., Takamine, F., and Uchida, K. (1999). Absence of cecal secondary bile acids in gnotobiotic mice associated with two human intestinal bacteria with the ability to dehydroxylate bile acids in vitro. Microbiol. Immunol. 43, 893-897. doi: 10.1111/j.1348-0421.1999.tb0 1224.x

Nitzan, O., Brodsky, Y., Edelstein, H., Hershko, D., Saliba, W., Keness, Y., et al. (2017). Microbiologic data in acute cholecystitis: ten years' experience from bile cultures obtained during percutaneous cholecystostomy. Surg. Infect. (Larchmt.) 18, 345-349. doi: 10.1089/sur.2016.232

Portela, C. A., Smart, K. F., Tumanov, S., Cook, G. M., and Villas-Boas, S. G. (2014). Global metabolic response of Enterococcus faecalis to oxygen. J. Bacteriol. 196, 2012-2022. doi: 10.1128/JB.01354- 13

Ridlon, J. M., Harris, S. C., Bhowmik, S., Kang, D. J., and Hylemon, P. B. (2016). Consequences of bile salt biotransformations by intestinal bacteria. Gut Microbes 7, 22-39. doi: 10.1080/19490976.2015.112 7483

Ridlon, J. M., Kang, D. J., and Hylemon, P. B. (2006). Bile salt biotransformations by human intestinal bacteria. J. Lipid Res. 47, 241-259. doi: 10.1194/jlr. R500013-JLR200

Rutherford, S. T., and Bassler, B. L. (2012). Bacterial quorum sensing: its role in virulence and possibilities for its control. Cold Spring Harb. Perspect. Med. 2:a012427. doi: 10.1101/cshperspect.a012427

Salvioli, G., Salati, R., Bondi, M., Fratalocchi, A., Sala, B. M., and Gibertini, A. (1982). Bile acid transformation by the intestinal flora and cholesterol saturation in bile. Effects of Streptococcus faecium administration. Digestion 23, 80-88. doi: 10.1159/000198691

Scherer, M., Gnewuch, C., Schmitz, G., and Liebisch, G. (2009). Rapid quantification of bile acids and their conjugates in serum by liquid chromatography-tandem mass spectrometry. J. Chromatogr. B Analyt. Technol. Biomed. Life Sci. 877, 3920-3925. doi: 10.1016/j.jchromb.2009.0 9.038

Seemann, T. (2014). Prokka: rapid prokaryotic genome annotation. Bioinformatics 30, 2068-2069. doi: 10.1093/bioinformatics/btu153

Segata, N., Bornigen, D., Morgan, X. C., and Huttenhower, C. (2013). PhyloPhlAn is a new method for improved phylogenetic and taxonomic placement of microbes. Nat. Commun. 4:2304. doi: 10.1038/ncomms 3304

Shapiro, H., Kolodziejczyk, A. A., Halstuch, D., and Elinav, E. (2018). Bile acids in glucose metabolism in health and disease. J. Exp. Med. 215, 383-396. doi: 10.1084/jem.20171965

Shen, H., Ye, F., Xie, L., Yang, J., Li, Z., Xu, P., et al. (2015). Metagenomic sequencing of bile from gallstone patients to identify different microbial community patterns and novel biliary bacteria. Sci. Rep. 5:17450. doi: 10.1038/ srep 17450

Song, X., Sun, X., Oh, S. F., Wu, M., Zhang, Y., Zheng, W., et al. (2020). Microbial bile acid metabolites modulate gut RORgamma(+) regulatory $\mathrm{T}$ cell homeostasis. Nature 577, 410-415. doi: 10.1038/s41586-019-1 865-0

Song, Z., Cai, Y., Lao, X., Wang, X., Lin, X., Cui, Y., et al. (2019). Taxonomic profiling and populational patterns of bacterial bile salt hydrolase (BSH) genes based on worldwide human gut microbiome. Microbiome 7:9. doi: 10.1186/ s40168-019-0628-3

Stewart, L., Griffiss, J. M., Jarvis, G. A., and Way, L. W. (2007). Gallstones containing bacteria are biofilms: bacterial slime production and ability to form pigment solids determines infection severity and bacteremia. J. Gastrointest. Surg. 11, 977-983; discussion983-974. doi: 10.1007/s11605-007-0 $168-1$

Tajeddin, E., Sherafat, S. J., Majidi, M. R., Alebouyeh, M., Alizadeh, A. H., and Zali, M. R. (2016). Association of diverse bacterial communities in human bile samples with biliary tract disorders: a survey using culture and polymerase chain reaction-denaturing gradient gel electrophoresis methods. Eur. J. Clin. Microbiol. Infect. Dis. 35, 1331-1339. doi: 10.1007/s10096-0162669-x

Thomas-White, K., Forster, S. C., Kumar, N., Van Kuiken, M., Putonti, C., Stares, M. D., et al. (2018). Culturing of female bladder bacteria reveals an interconnected urogenital microbiota. Nat. Commun. 9:1557. doi: 10.1038/ s41467-018-03968-5

Tidjani Alou, M., Naud, S., Khelaifia, S., Bonnet, M., Lagier, J. C., and Raoult, D. (2020). State of the art in the culture of the human microbiota: new interests and strategies. Clin. Microbiol. Rev. 34:e00129-19. doi: 10.1128/CMR.001 29-19

Wadhwa, V., Jobanputra, Y., Garg, S. K., Patwardhan, S., Mehta, D., and Sanaka, M. R. (2017). Nationwide trends of hospital admissions for acute cholecystitis in the United States. Gastroenterol. Rep. (Oxf.) 5, 36-42. doi: 10.1093/gastro/ gov015

Wang, B. F., Yi, S., Keshavamurthy, J., Williams, H., and Pucar, D. (2021). Gallbladder perforation into the peritoneal cavity. Clin. Nucl. Med. 46, e84-e85. doi: 10.1097/RLU.0000000000003317

Wang, J., and Jia, H. (2016). Metagenome-wide association studies: fine-mining the microbiome. Nat. Rev. Microbiol. 14, 508-522. doi: 10.1038/nrmicro.20 16.83

White, B. A., Lipsky, R. L., Fricke, R. J., and Hylemon, P. B. (1980). Bile acid induction specificity of 7 alpha-dehydroxylase activity in an intestinal Eubacterium species. Steroids 35, 103-109. doi: 10.1016/0039-128x(80)90115-4

Woo, P. C., Fung, A. M., Lau, S. K., and Yuen, K. Y. (2002). Identification by 16S rRNA gene sequencing of Lactobacillus salivarius bacteremic cholecystitis. J. Clin. Microbiol. 40, 265-267. doi: 10.1128/jcm.40.1.265-267.2002

Wu, T., Zhang, Z., Liu, B., Hou, D., Liang, Y., Zhang, J., et al. (2013). Gut microbiota dysbiosis and bacterial community assembly associated with cholesterol gallstones in large-scale study. BMC Genomics 14:669. doi: 10.1186/ 1471-2164-14-669 
Yang, J., and Yu, J. (2018). The association of diet, gut microbiota and colorectal cancer: what we eat may imply what we get. Protein Cell 9, 474-487. doi: 10.1007/s13238-018-0543-6

Yun, S. P., and Seo, H. I. (2018). Clinical aspects of bile culture in patients undergoing laparoscopic cholecystectomy. Medicine (Baltimore) 97:e11234. doi: 10.1097/MD.0000000000011234

Zankari, E., Hasman, H., Cosentino, S., Vestergaard, M., Rasmussen, S., Lund, O., et al. (2012). Identification of acquired antimicrobial resistance genes. J. Antimicrob. Chemother. 67, 2640-2644. doi: 10.1093/jac/dk s261

Zheng, B., Jiang, X., Cheng, H., Guo, L., Zhang, J., Xu, H., et al. (2017). Genome characterization of two bile-isolated Vibrio fluvialis strains: an insight into pathogenicity and bile salt adaption. Sci. Rep. 7:11827. doi: 10.1038/s41598-01712304-8

Zou, Y., Xue, W., Luo, G., Deng, Z., Qin, P., Guo, R., et al. (2019). 1,520 reference genomes from cultivated human gut bacteria enable functional microbiome analyses. Nat. Biotechnol. 37, 179-185. doi: 10.1038/s41587-018-0008-8
Conflict of Interest: The authors declare that the research was conducted in the absence of any commercial or financial relationships that could be construed as a potential conflict of interest.

Publisher's Note: All claims expressed in this article are solely those of the authors and do not necessarily represent those of their affiliated organizations, or those of the publisher, the editors and the reviewers. Any product that may be evaluated in this article, or claim that may be made by its manufacturer, is not guaranteed or endorsed by the publisher.

Copyright (c) 2021 Yan, Zhang, Li, Wang, Zhang, Jin, Zhang, Lv, Xiao, Sun, Li, Cui, Li, Ma, Wang, Tian, Duan, Xin, Mao and Ma. This is an open-access article distributed under the terms of the Creative Commons Attribution License (CC BY). The use, distribution or reproduction in other forums is permitted, provided the original author(s) and the copyright owner(s) are credited and that the original publication in this journal is cited, in accordance with accepted academic practice. No use, distribution or reproduction is permitted which does not comply with these terms. 